\title{
Scoliosis and Osteopathy
}

\section{René Zweedijk*, Christophe Tylleman and Peter Schwind}

Department of Osteopathic Education, Pro Osteo Privat Teaching Institution, The

Netherlands

*Corresponding Author: René Zweedijk, Department of Osteopathic

Education, Pro Osteo Privat Teaching Institution, The Netherlands.
Received: August 05, 2020

Published: August 24, 2020

(C) All rights are reserved by René Zweedijk., et al.

\begin{abstract}
Scoliosis is a frequently seen problem in the osteopathic practice. There are different types of scoliosis. The most disabling form is the Adolescent Idiopathic Scoliosis (AIS). Humans are chaotic, complex adaptive systems. The consequence is that many different factors can cause AIS and that the onset can be sudden and quick. In this article we study the etiology of AIS by using the 2012 SOPE model of 5 of osteopathy. AIS has mechanical, neurological, respiratory, circulatory, metabolic and bio-psychosocial aspects. It also means that the osteopath should see every patient as unique and analyze all factors that are putative causal in the onset of AIS. Some treatment options are shown based on recent scientific insights.
\end{abstract}

Keywords: Scoliosis; Osteopathy; Adolescent Idiopathic Scoliosis (AIS)

\section{Introduction}

Scoliosis can be a very disabling change in form and function and is defined as a three-dimensional deformation of the spine. It is considered to have a multi-factorial aetiology with factors including genetic predisposition, imbalance between anterior and posterior spinal growth and abnormalities in connective tissue, skeletal muscles and nerves [1]. The condition occurs in approximately $3 \%$ of the population but mostly girls (sex ratio 8:1) [2]. Compared to the number of articles about the treatment of scoliosis, there are fewer articles about its aetiology and pathology [3]. It is remarkable that A.T. Still did not mention scoliosis in his works. The only thing he mentioned was a lateral curvature of the spine caused by joint dysfunctions. John Wernham, who continued the work of Martin Littlejohn, concentrated on the correction of the curves and the mobility of key vertebra in the spine. In the later works of Chilla and Ward, the description of scoliosis is not different from the descriptions found in classical works in allopathy $[4,5]$ In the works of Sutherland, a scoliosis was related to a cranial dysfunction [6]. Barral describes the relation between a visceral dysfunction and a spinal displacement [7]. As in the classical articles, in the osteopathic works the aetiology of scoliosis is not well described. Of course, the osteopath treats what he finds. However, more knowledge of the aetiology will improve the effect of the treatment. In this article, we describe the types of scoliosis and we elucidate the aetiology of scoliosis and describe some possibilities of the osteopathic treatment.

\section{Classification of scoliosis}

There are several ways in which to classify scoliosis. A first method is to distinguish between a structural and a functional form. With functional or postural scoliosis, there is no change in the structure of the bones and there is no weakness of the muscles. The main causative factor in this form is incorrect posture, caused by fascial, muscular or postural reflex mechanisms. In time, this can result in secondary restrictions in the soft tissues [8].

The second type of scoliosis is the structural scoliosis. In the structural form of scoliosis, there is a bone deformation in combination with restrictions in the soft tissues. 
Another classification of scoliosis is the classification according to its aetiology: paralytic, idiopathic or congenital. It can be further classified as infantile ( 0 to 3 years), juvenile (3 to 10 years), adolescent (10 to 18 years) or adult (above 18 years) [3].

Paralytic scoliosis is a result of a muscular or neurological disease. The idiopathic form in children in the age of 10-18 years old, named Adolescent Idiopathic Scoliosis (AIS) is the most common form of scoliosis.

Congenital scoliosis develops while a baby is in the womb. Sometimes it can go unnoticed until a child goes through a growth spurt, for example around the age of two years, or during the early teenage years. In the latter case, congenital scoliosis may resemble AIS.

AIS is the least understood but the most interesting form of scoliosis. A remarkable aspect is the speed with which it develops. Once the scoliosis is structural, it is hard to influence. Therefore osteopaths need to know more about the aetiological mechanisms in order to be able to treat it as early as possible in order to prevent the scoliosis from becoming structural.

In this article, we will mainly concentrate on AIS. Of course, the other forms of scoliosis may be related to AIS.

In AIS, there are four common types of curve patterns [9]:

- $\quad$ Thoracic ( $90 \%$ on the right side),

- Lumbar (70\% on the left side)

- Thoracolumbar (80\% on the left side) and double curved scoliosis (both on the left and right side).

The Cobb method is the most commonly used method to describe the gradation of scoliosis. For the Cobb method, it is necessary to determine the end vertebrae, which are situated at the upper and lower ends of the curve and which tilt the most towards the concave of the curve. Lines are drawn along the upper endplate of the upper body and along the lower endplate of the lower body. The angle formed at the intersection of these lines is known as Cobb's angle [10].

\section{Model of 5}

In 2012, the EFO (European Federation of Osteopaths) presented a document about the scope of osteopathic practice in Europe
(SOPE). SOPE is the most up-to-date model, underpinning current osteopathy. As stated in SOPE, osteopathy focuses on promoting the ability of the body to recover by itself and in so doing relies upon the concept of the unity of the individual's structure (anatomy) and function (physiology). The human body is a unit formed by the permanent continuity of structures. The most striking continuity is that of the fascial system. However, the vascular system, the nervous system, the immune system are also responsible for the unity of the human body. A dysfunction in the body disrupts the normal physiological functioning of the structure in question and involves a disturbance of the mechanisms, which attempt to maintain normal homeostatic and allostatic equilibrium [11].

A dysfunction involves the loss of normal mobility. This loss of mobility is the common factor within the following 5 aspects/models:

\section{- Mechanical model}

- Neurological model

- Respiratory circulatory model

- Bioenergetic/metabolic model

- Bio-psychosocial model.

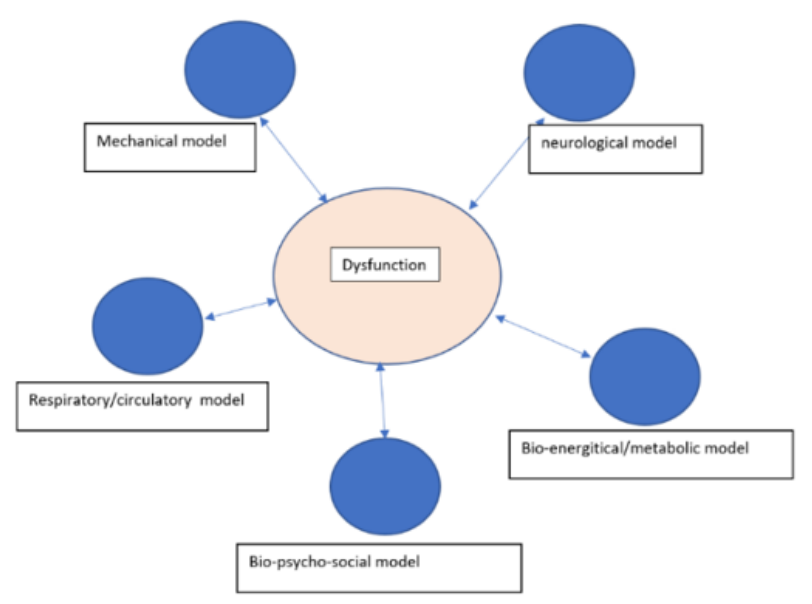

Figure 1

Within the mechanical model, it is clear that a structure must move in order to function properly. The mechanical model is applicable in the musculoskeletal system, the visceral and cranial sys- 
tem. Within the neurological model, a dysfunction involves the loss of mobility and leads to a disturbed balance between input and output of the nervous system. The nervous system can be damaged at various levels, resulting in the self-healing force of the body being disturbed. The loss of mobility of the nervous system in itself falls under the mechanical model.

Disruption of the respiratory system and disturbance of blood flow are seen as dysfunctions of the respiratory-circulatory model. According to the arterial rule, Still emphasizes the importance of proper circulation of all body fluids.

It is more difficult to describe a dysfunction in the bioenergetical model. The general point is that when the mobility of structures is disturbed, this influences the metabolic processes related to that structure and this is reflected in a decrease in vitality. In addition to the fact that it concerns the spine, a decrease in the ability of the neck to move will also indirectly affect metabolism in the thyroid gland. This is therefore a distortion at a bioenergetic/metabolic level. Dysfunctions at endocrine level and in the immune systems are also covered by this model.

A human being, and most certainly a child, is in constant contact with his surroundings. Processes in the environment affect the welfare of the child and his ability to recover. Stress, abuse, traumata and the like are dysfunctions that fall under the bio-psychosocial model. The loss of mobility and its restoration through manual treatment is of central importance within all models.

\section{Model of 5 and scoliosis}

The osteopathic analysis of a scoliosis is characterized by an analysis of the applicability of the different models mentioned above. The mechanical model focuses on the loss of flexibility within the musculoskeletal system, the fascial system, the visceral system (a part of the fascial system) and the cranium. Dysfunctions in these areas can lead people to develop a scoliosis. The neurological model focuses on the role of the ANS (autonomic nervous system) and the somatic nervous system in the creation of scoliosis. Within the respiratory system, we discuss the role of the aperture in the creation of scoliosis. The circulation system, however, focuses on the role of SCF (cerebrospinal fluid) and of the arterial and venous system. In the bioenergetical/metabolic model, we discuss the influence of the hormonal system and finally the bio-psycho- social model highlights the influence of the mind on the creation of scoliosis.

\section{Mechanical model}

Early osteopaths maintain that an infantile scoliosis which arises early (between 0 and 3 years) is not the result of gravity during walking [12]. Rather it has to do with forces that have worked on the body during pregnancy, birth or the postnatal period. The cranium in early development has several synchondroses, which ossify in older children. Also, the sutures in younger children are much more mobile because they are still in development. Dysfunctions at the level of the synchondrosis and the sutures but also patterns of strain upon the membranes can affect the emergence of the scoliosis at the level of the cranium. In particular, the sphenobasilar synchondrosis and the occiput appear to play an important role in the creation of a cranial scoliosis [13]. Nakashima demonstrated in a study of 79 patients that there is a positive correlation between a scoliosis and a displacement of the mandibula [14]. Kim found no correlation in his study between the severity of an idiopathic scoliosis and the degree of scoliosis at the height of frontal and lateral movements in the face [15]. The ossification of the neurocranium is earlier compared to the viscerocranium. The face has its greatest growth when the child changes the milk dentures for fixed teeth while the neurocranium has its biggest growth spurt in the years before this. In practice, in children and adults with a distorted "scoliotic" skull, lateral strain with SB rotation pattern can be found both in the neurocranium and in the viscerocranium. The cause of the scoliosis should be sought in the period when growth is still taking place in the two parts of the skull, i.e. in the period of pregnancy, birth and the period thereafter. When the scoliosis can only be found in the face and not in the neurocranium, the scoliosis has arisen in the period of strong growth, i.e. after 5-6 years. One finds a cranial base without strain pattern but a strain pattern in the face. Figure (below) shows a man with a fibrosis of the sternocleidomastoideus muscle caused by a trauma during birth. The skull base has a light strain patterns in this man, but there is a very strong asymmetry in the face that has even distorted both temporals into intraosseous. In the photos taken during the first few years, there is no or hardly any visible pathology while a clear strain image can be seen in the third photo (end of primary school). Moreover, the person in the image does not have spinal scoliosis, indicating that this is not a prerequisite even though there is a positive statistical relationship between a scoliosis of the face and a spinal scoliosis [15]. 


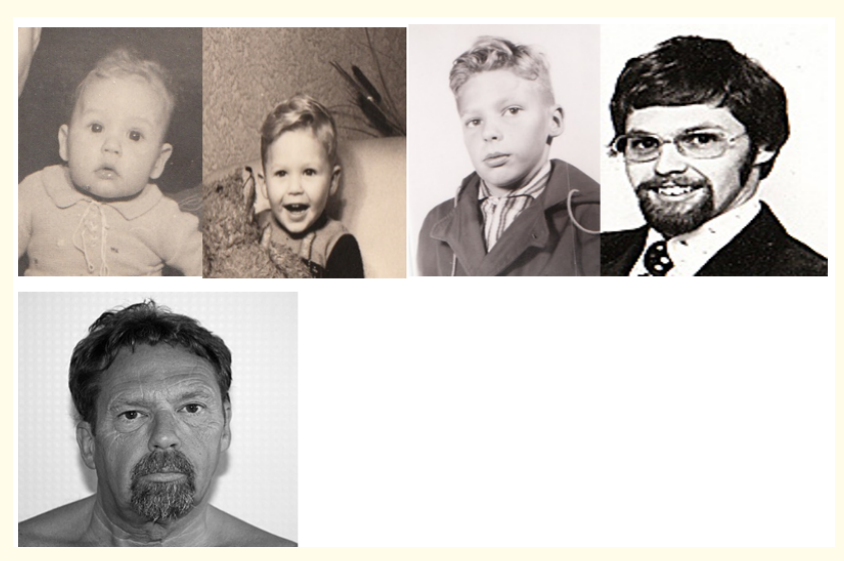

Figure 2: Demonstrates the evolution of a cranial scoliosis. At the photo is at the age of 6 month, 2 years, 11 years, 23 years and 68 years. Note that the torticollis is present at birth, that there is hardly a scoliosis visible but after the development of the face(11 years) there is a strong facial scoliosis that stays lifelong.(photo's with permission of patient, Rene Zweedijk).

Uncoupled neuro-osseous growth along the longitudinal axis of the spinal cord is described as a possible cause for AIS. Roth (1968), a neuroradiologist, was the first to report a short spinal cord in idiopathic scoliosis [16]. Porter confirmed this as the pathogenesis of AIS. This is described in literature as the Roth-Porter pathogenetic hypothesis of disproportionate growth between the spinal cord and the vertebral column [17]. More recent studies have confirmed this theory [10].

Anteriorly the vertebral bodies grow faster than the posterior elements, which results primarily in a lordosis (or hypokyphosis). The diminished dorsal growth impedes the ventrally located vertebral bodies from increasing in height, forcing them to become distorted (rotate), in order to create space for themselves. The flattening or more usually reversal of the normal thoracic kyphosis at the apex of the scoliosis or asymmetry in the median plane is crucial in the development of a progressive AIS. This is because many normal children have coronal plane asymmetry and all have vertebral body asymmetry in the transverse plane.

Guo found that scoliotic spines had longer vertebral bodies between T1 and T12 in the anterior column and shorter pedicles with a larger interpedicular distance in the posterior column
[18]. The differential growth between anterior and the posterior elements of each thoracic vertebra in the patients with AIS was significantly different from that in the controls.

Disproportionate neuro-osseous growth might explain why the apex of scoliosis is frequently in the lower thoracic spine. The vertebral epiphyses close earlier in the upper region than in the lower thoracic spine and the major component of adolescent longitudinal spinal growth takes place in the lower thoracic spine between $\mathrm{T} 5$ and $\mathrm{T} 10$. This part of the spine would be most vulnerable to deformity if at that time there was a disproportionate growth.

Chu found in MRI a significant relative segmental lengthening of the spinal column at the thoracic level in AIS patients with severe curve [19]. This suggests a disproportional growth between the skeletal and the neural systems. Their conclusion was that the relative shortening and functional tethering of the spinal cord may play an important role in the pathogenesis of AIS [20].

\section{Filum terminale syndrome or cord traction syndrome and AIS}

The filum terminale is classically described as a fibrous band connecting the conus medullaris with the coccyx. The cranial and intradural part is called the filum terminale internum (FTI), which connects the conus medularis and the distal termination of the dural sac. The caudal extradural part is the filum terminale externum (FTE), also called the lig. Coccygeale. This connects the distal culde-sac and the periosteum of the coccyx. Recent histological and embryological studies [21] show that describing the filum terminale as a fibrous band is an oversimplification. The filum terminale does not perforate or pierce through the dural sac. Instead the dura fuses with the FTI and continues further as FTE.

The filum terminale with its meningeal coverings emerges below the sacral hiatus and passes downwards across the dorsal surface of S5 and the sacro-coccygeal joint to reach the coccyx, with the first coccygeal nerve running along the filum. The filum is encircled by a connective tissue sheath, which is continuous with the pia mater at higher levels. The denticulate ligament, which extends as a narrow band along the lateral aspect of the pia mater, becomes continuous with the sheath of the filum. The filum terminale has, along with the denticulate ligaments, the capacity of protecting the spinal cord against physiological stress [21]. Elasticity tests on living cats and fresh human cadavers shows also that the filum buffers elongation stress on the spinal cord. 
Section of the filum terminale has been shown to be a useful strategy in the treatment of scoliosis, syringomyelia and the Chiari malformation, which indicates the importance of this structure in the aetiology of these pathologies [22].

The relative shortening and functional tethering of the spinal cord may play an important role in the pathogenesis of AIS. Royo-Salvador found that AIS shares the same aetiopathogenic mechanism as syringomyelia and Arnold-Chiari malformations an abnormal lack of synchronization of the growth of the neuro-axis and the neural canal. In the tethered cord syndrome, the spinal cord is pulled downwards [23]. One of the reasons for this can be a tight filum terminale.

An understanding of neural tube development is the key in understanding, diagnosing and treating tethered cord syndrome. In early embryonic life, the spinal cord occupies the entire length of the vertebral canal. When an embryo reaches $30 \mathrm{~mm}$ in length, around the second trimester, there is an increasing discrepancy between the anatomical level of the vertebral column and the spinal cord, the former elongating more rapidly than the latter. By full term, near 40 weeks, the conus medullaris lies between the first and third lumbar vertebrae. Patients more than 3 months of age and definitely those more than 6 months of age with a conus found below the mid-body of L2 should be evaluated. This situation is described as 'filum terminale or cord traction syndrome'; this structure is responsible for the tethered cord.

\section{Neurological model and scoliosis}

The nervous system possesses a large amount of asymmetry. The fact that the cortex of both hemispheres largely differ in function is well known. However, there are other differences between the right and left hemispheres. In the development of scoliosis, the nervous system can play a major role. Faloon showed that in $8 \%$ of scoliosis, there are neuroaxial deformities (Chiari malformations and Syringo formation), which is a high percentage [24].

The influence of the brain on the onset of scoliosis is associated with the function of the autonomic nervous system, the hormonal system, as well as with the function of the somatic nervous system. Craig has demonstrated that the autonomic nervous system in the periphery knows a strong asymmetry [25]. This is, for example, visible in the control of the heart action, especially its rhythm, which is regulated mainly from the right side. In addition, Craig

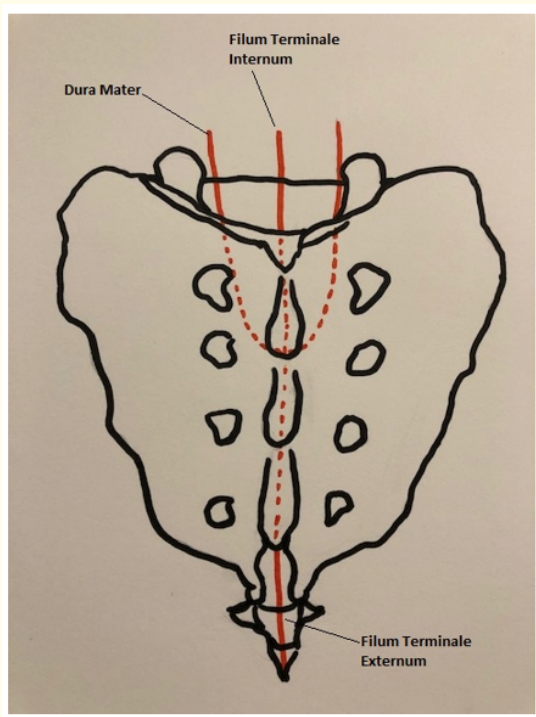

Figure 3: Demonstrates the external and internal Filum Terminale. Note that the dura end at the level of S2 but that the filum continues and ends at the CX.

indicates that the central nervous system also has a large asymmetry. The asymmetry is found at the level of the hypothalamus, the insular cortex, the amygdala, hippocampus and the frontal cortex. In the case of resting, a trophotrope function, the left hemisphere appears to be active, a reason for Craig to describe it as parasympathetic, sometimes also called vagal [25]. The right hemisphere is more active during active/ergotrope functions and is therefore considered to be more sympathetic. Stress and relaxation thus have a direct influence on the asymmetry of the brain and thereby influence the somatic and autonomic nervous system, with scoliosis as a possible consequence.

Somatic nervous system: Burwell describes the somatic nervous system escalator concept. The sensory information from the proprioceptive pelvic floor and interoceptive system is integrated with the descending motor influence. In circumstances where there is an asymmetry in the activity of the brain, this leads to an asymmetrical regulation of the postural aspects of the axial skeleton, resulting in a scoliosis [26].

Autonomic nervous system: the autonomic nervous system is strongly influence by the hypothalamus. The hypothalamus regu- 
lates both the autonomic nervous system as well as the hormonal system in which the sensitivity of the hypothalamus is regulated by circulating hormones and other factors. Leptin appears to be an important hormone, which plays a role in the onset of scoliosis and directly influences the sensitivity of the hypothalamus. This increased sensitivity in combination with the asymmetry described by Craig can lead to asymmetric "steering" of the peripheral autonomous function [25]. There is a tendency that the right side of the brain is dominant. This side influences the sympathetic part of the nervous system and the result is that the right side of the body is more sympathetically active (SNS: Sympathetic Nervous System). Keep in mind that autonomous nerve fibres do NOT change sides as motor fibres do. The growth disks at the height of the axial skeleton, the ribs, the pelvis and the clavicle are all strongly influenced by the SNS. This is in contrast to the growth disks in the periphery of arms and legs. A disturbed sympathetic regulation of the growth leads to an asymmetry at the height and or length of the clavicle, the rips and pelvis but not to an asymmetry in the extremities [27].

A scoliosis (AIS) can be the result of asymmetrical activity in central parts of the brain. As described in the literature, this can be the result of, for example, stress or increased physical activity as in sport. It should be noted that the SNS is directly in contact with the hormonal system and that, for example, growth hormone from the pituitary gland has an important function in the growth of the skeleton.

An interesting idea was published by Morningstar [28]. In his view, children with AIS have a different status of neurotransmitter networks in the brain. Morningstar describes the influence of serotonin, adrenalin, noradrenaline and histamine. He proposes that an imbalance in concentration of these neurotransmitters and a changed activity of the networks that produce these neurotransmitters influence postural control and can influence the onset of AIS.

\section{Respiratory and circulatory aspects}

Much research has been done on the influence of circulatory aspects in the onset of scoliosis. CSF (Cerebrospinal Fluid) Circulation and the arterial system appear to play a role in the development of scoliosis, in particular of AIS.

\section{LCS circulation}

Grimes describes the influence of CSF circulation and the emergence of a scoliosis in zebra fish [29]. PTK 7 Mutant zebra fish have a defect in the development of the cilii, which are responsible for the circulation of CSF in the spinal canal. These fish develop a scoliosis (see image below). When the cilii recover, the development of the scoliosis ceases. Grimes therefore concludes that the CSF circulation, which depends on the cilii movement, triggers an irregular circulation of CSF. This can then be an underlying biological cause for the development of an AIS.

\section{Venous system}

Talic describes a high incidence of varices in people with AIS [30]. He ascribes this to increased slackness of the tissues and not so much to a changed venous circulation. Grant describes a large variation in the anatomy of the basal vertebral veins. However, there is no difference between healthy individuals and people with AIS. There is therefore no valid evidence for an influence of the venous system on the development of a scoliosis, even on AIS [31].

\section{Arterial system}

The arterial system appears to have a greater influence on the development of a scoliosis, especially on AIS. As early as 1982, Ratcliffe described the importance of the arteries in the development of scoliosis [32]. In children, there is an extensive anatomic network of intra-osseous vessels. At the age of 7, this network begins to change and at the age of 15 the network reaches its adult form. According to Ratcliffe, this change is one of the causes of the emergence of AIS. An uneven speed of obliteration at different sides of the vertebral column can play a role here.

$90 \%$ of the high thoracic scolioses are right convex. In Cardiodextra, this is not the case and a left convex scoliosis is seen. The convexity of the scoliosis is thus influenced by the organ orientation, in this case the heart and the aorta. Hua Jiang describes an altered aortic position in people with AIS [33]. It is not clear whether this is a change in the blood vessel caused by the scoliosis or whether the altered blood vessel position affects the scoliosis. Rajiv Kaila describes a scoliosis in a monozygotic twin, which disappeared after an arterial pulmonary sleeve was cut [34]. This indicates that there are clear influences related to the heart and the large vessels. Sevastik describes the importance of a long arteria intercostalis on the right side in the emergence of a right convex scoliosis [35].

\section{Bone marrow and embryonic development}

The bone marrow is the basis of both the arterial system and the immune system. Mechanical forces are important in the emer- 
gence of the skeleton during embryonic development and during later growth. Mechanical forces affect the growth of bone cells that originate in the mesoderm. Levillain describes that a short time of foetal immobility can affect spinal curvature and anatomy in chickens [36]. Zaydman describes that neural list cells can move to the concave side of the scoliosis during embryonic development where they can disturb the growth of the vertebral body on that side. This may be an important factor in the development of AIS [37].

\section{Metabolic/bio-energetic model and scoliosis}

The influence of the hormonal system on the onset of scoliosis has been known for a long time. The fact that girls develop a scoliosis more frequently during the period of puberty indicates that there must be a strong hormonal influence. In the following, we discuss some of the hormones involved.

\section{Epiphysis and melatonin}

As early as 1959, Thillard showed that damage to the epiphysis in chickens caused a scoliosis. This was confirmed by Dubousset. The study also indicated that intra-peritoneal injections protected the hens from developing a scoliosis [38].

A study by Kaustubh shows that children with idiopathic scoliosis have a lower amount of melatonin in their blood, compared to children without scoliosis and to children with congenital scoliosis [39]. It also appears that in the group with the idiopathic scoliosis the amount of parathyroid hormone, Vitamin D and alkaline phosphatase, are lowered. These deficiencies indicate a low mineral density, an increased "bone-turnover" and a negative calcium balance.

A striking fact is that the epiphysis is the only structure that is not present in both sides of the brain and is also the only real midline structure in the body. This makes it for author one of the most important structures in the brain for osteopathy. Some studies have also indicated the importance of Calmoduline, an antagonist of melatonin [40]. Calmoduline, which works among other things on the contractility of smooth muscle fibres, is increased in the paraspinal musculature at the level of the convexity of the curve in scoliosis. It is believed, however, that it does not play a role in the aetiology of scoliosis but is an indication for changes in local calcium metabolism.
Leptine

Burwell describes a lower body weight, a lower fat-free mass, a lower quantity of freely leptin in blood and a high adiponectin in 10-year-old girls, who developed a scoliosis at 15 years. He also describes a higher incidence of scoliosis in both low-BMI girls and girls with high BMI. The incidence of AIS in low BMI is highest. The altered leptin production and altered leptin sensitivity of the hypothalamus, as is also described in overweight, appears to play a role in the emergence of an idiopathic scoliosis. Burwell speaks of the leptin-hypothalamus-sympathetic axis [27].

\section{Other hormones}

Other hormones also play an important role. Growth hormone has been studied intensely and appears to be elevated in children with idiopathic scoliosis; FSH, LH and estradiol are lowered. Oestrone, estriol, progesterone and testosterone are higher in children with scoliosis. The mechanism of this is not yet totally understood [40].

\section{The gut}

Shen observed a significant, positive correlation between the abundance of the faecal genera Prevotella and the Cobb angles of the Chinese AIS patients [41]. This could indicate that a disturbed digestive function with changes in the microbiom could affect the onset of AIS.

\section{Environmental factors}

The following environmental factors are known to affect the onset of idiopathic scoliosis: stress, alcohol consumption, nutrition, smoking, viruses, drug use, medication, toxins, physical activity. High levels of copper (Cu), zinc ( $\mathrm{Zn}$ ) and low levels of selenium (Se) appear to play a role, as well as Vitamin D and blood calcium levels [40]. In swimmers, there is a higher incidence of scoliosis and this may be due to exposure to the air in swimming pools. A high concentration of chloride provoked mice to develop scoliosis. This may be one of the reasons, apart from low body weight, stress and hypokyphosis, that swimmers develop more scoliosis as compared with other sports.

\section{Bio psychosocial aspects of scoliosis}

In the models that have been presented above, it is clear that stress is an important factor in the onset of AIS by causing asymmetrical stimulation of the ANS and the somatic nervous system. 
Burwell suggested that scoliosis is caused by environmental stress, leading to developmental instability [27]. Environmental factors could be hormonal, nutritional, alcohol, smoking, viruses, drugs, medications, toxins, and physical activity. Additionally, several authors have noted that scoliosis occurs in children in response to psychological distress, trauma, back injury, surgery, cancer treatment (radiation and chemotherapy), infections, tumours and injuries sustained at birth [40].

Hobbies and sports seem also to have an influence on the incidence of AIS. Adolescent ballet dancers are at significantly higher risk of developing scoliosis than non-dancers of the same age. Odds ratio calculations suggest that dancers are 12,4 times more likely to develop scoliosis than non-dancers of the same age. Girls, who do intense swimming training also have a higher incidence of AIS [42]. Rhythmic gymnasts have a 10-fold higher incidence of scoliosis than a control group. But there is no correlation between AIS and exercising because no statistically significant difference has been found between athletes and non-athletes. One study, however, does suggest that there is a 'dangerous triad' of generalized joint laxity, delayed maturity and asymmetrical spinal loading in gymnasts [42].

\section{Genetic and epigenetic aspects of the onset of AIS}

Ogura and others have performed genome-wide association studies (GWASs) and identified several loci associated with AIS susceptibility (occurrence) [43]. Genomic studies of susceptibility to AIS have been revealing. Ogura identified a locus associated with AIS curve progression with convincing evidence. In clinical practice, however, the value of AIS susceptibility loci and genes is limited [43]. Kikanloo stated that several genes are important for the onset of AIS [44]. These genes influence the production of peptides like calmodulin and influence bone development, elasticity of tissues and paraspinal muscle development [44].

Meng., et al. reported in EBioMedicine, a genome-wide methylation analysis for curve progression of adolescent idiopathic scoliosis (AIS) [45]. They examined DNA methylation differences between disconcordant monozygotic (MZ) twins and found decreased methylation at cg01374129 (epigen) associated with AIS curve progression. This is the first large-scale epigenetic study for AIS and its progression. Further epigenetic analyses, such as histone modifications and chromatin accessibility and their combination with other omic studies such as RNA-seq, would be necessary to secure the ultimate goal of developing an accurate prognostic test for AIS [46].

These findings are important for osteopaths. An early diagnosis and treatment of factors that are significant in the aetiology of AIS, before the AIS becomes structural, will possibly influence methylation, histone modification and chromatin accessibility that are responsible for the development of AIS.

\section{Chaotic systems, complex adaptive systems and scoliosis}

It is important to keep in mind when diagnosing, but also during treatment, that a human being is not a machine. Human beings qualify to be seen as chaotic systems. This implies that different conditions can lead to scoliosis, but also that scoliosis in one patient may need a different approach from that appropriate in a different case. When different people with scoliosis are treated with a similar approach, the improvement pathways will never be exactly the same, even if they may show similarities.

It is typical of chaotic systems that they exhibit chaotic behaviour but that there is also a certain amount of order. Chaos is therefore not complete disorder; chaos is concerned with the area between complete order and complete disorder. If there is too much chaos, then a system derails; if there is too much order, then it becomes too rigid. Life exits on the edge of chaos; that is, in a situation where life has enough stability to sustain itself and enough creativity to deserve the name of life. It is a constantly shifting battle zone between stagnation and anarchy, the one place where a complex system can be spontaneous, adaptive, and alive. Chaotic behaviour can be seen in systems with a positive feedback mechanism, which exaggerates the effects of even a small disturbance of the system. With a chaotic system, a small perturbation can have a very strong significant effect after a relatively long period in which nothing happened. There may be various factors which can provoke such a change [47]. Positive feedback plays a role in what is called co-evolution, which means that organisms create their environment and are in turn are themselves moulded by that environment.

For diagnosis and treatment, it is also important to realize that humans are not only chaotic but also complex adaptive systems. They adapt to changes in their body and the environment (homeostasis and allostasis) to maintain their ability to survive. They can adapt because they possess negative feedback mechanisms, which ensure that changes in one direction lead to adjustment in the 
other. Most importantly, complex systems theory shows that relationships between parts (e.g. cells, tissues, organ systems) lead to collective behaviour. The systems react as one, as a whole. Complex systems are self-organizing and there is no hierarchy of command and control. Complex adaptive systems have emergent properties, which do not react as the properties of the parts tend to react but demonstrate non-linear behaviour.

Chaos theory and complex systems theory make it clear that big changes in a chaordic organisation can take place very quickly, as clearly can be seen in the onset of AIS. The development of AIS is often very fast. Furthermore, one must realize that many causes can be found. The problem is purely individual (every patient is different) and it is a dynamic process. Recent studies, which prove that epigenetic factors are important in the onset of AIS, support this theory. The osteopathic approach should always be systemic and include all the aspects that can influence a human being. Treatment must be aimed at the self-organizing properties of the client. It must also be as early and preventive as possible because as soon as the scoliosis is structural, it is hard to influence [48].

\section{Multifactorial onset of AIS}

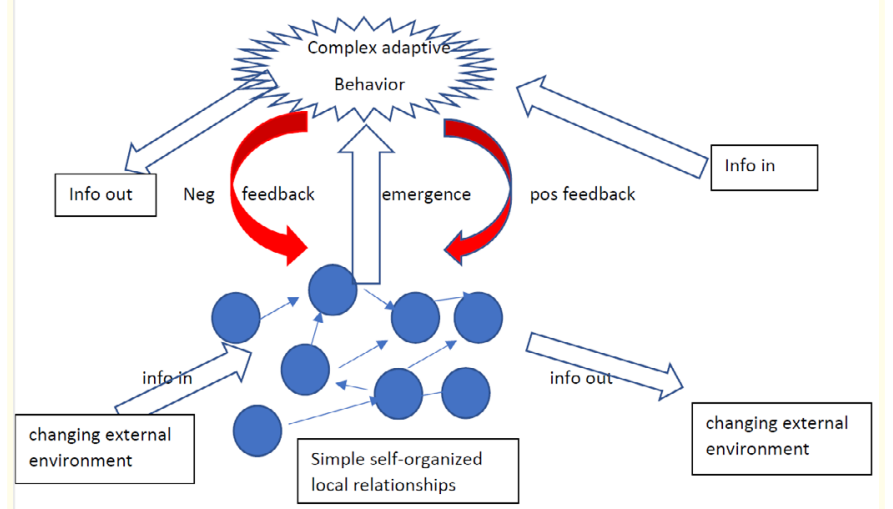

Figure 4: Complex adaptive system.

Osteopathic treatment of the scoliosis

Here we elucidate some of the possibilities for osteopathic treatment. It is not possible here to discuss all treatment options. We concentrate on personal insights and insights that are based on aetiological aspects. We concentrate on the treatment of AIS

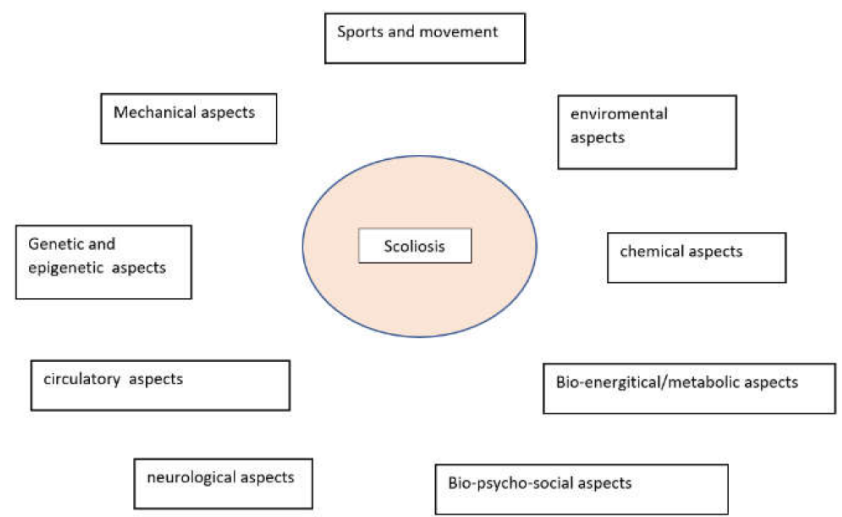

Figure 5

but the techniques can also be used for other forms of scoliosis (e.g. juvenile or adult scoliosis).

Special aspects of the three-dimensional arrangement of the fascial system and the correlation to the anatomical topography of the organs

When we evaluate the different manifestations of scoliosis from a three dimensional - a "volumetric" perspective - it becomes obvious that there is a clear relationship between the anatomical topography of the organs and the curves of the spine [27]. Embryology has documented the fact that different organs have to take a genetically well-defined "journey" during the early life of the embryo. During this journey, the organs have to find their place inside the cavities of the trunk. We may consider any deviation of the appropriate topography of an organ that appears in early childhood as one of the significant aspects of certain types of scoliosis. This seems to be relevant, especially in cases of unilateral displacement of the sub-diaphragmatic organs. If the stomach is positioned towards the midline and shows strong fascial connections towards the capsule of the liver, this will have an influence upon the three-dimensional activity of the respiratory diaphragm. The diaphragm then does not find the support of the stomach on the left side of the organism during exhalation. We will find a similar situation in cases in which the liver has shifted from the right towards the midline: the right side of the respiratory diaphragm will miss the support of the liver during exhalation. The underlying biomechanical mechanism becomes 
obvious if we consider that the hydrostatic pressure is significantly higher inside the peritoneum compared to the intra-thoracic space above the diaphragm.

We hypothesize that any irregular position of an organ is caused by an incomplete "journey" during the early life of the embryo. In clinical practice, we observed that the displacement of the stomach is quite frequent. The correct positioning of the stomach during early embryonic life starts with a rotation of 90 degrees around a vertical axis and ends with a tilt to the left that places the organ inside the peritoneum. Our hypothesis will need to find support by ultrasound documentation of the organ topography in the newborn until the end of puberty. Some information for validation may also come from documentation of observations, collected during abdominal surgery.

The above hypothesis may not only explain the position of single organs. It may also clarify the three-dimensional relationship and interaction between the intra-peritoneal and retro-peritoneal spaces. It may also elucidate the transmission of forces between several organs. This has to be explored in greater detail for the lungs and the heart.

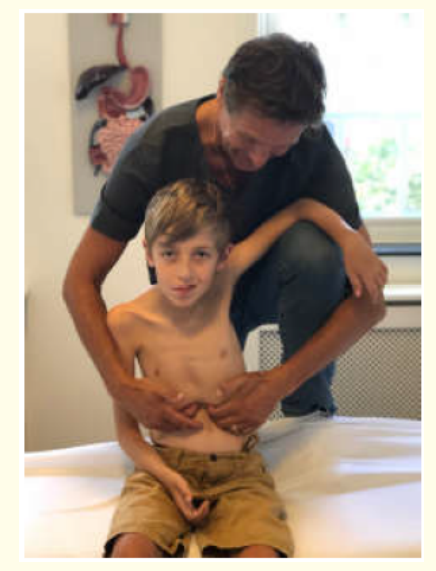

Figure 6: Illustration: The treatment of the diaphragm area in young patient with scoliosis[49].

\section{Mechanics of the nervous system: A functional continuum}

Looking at the nervous system in a mechanical way is not new. A. Breig established the concept of 'adverse mechanical tension'. In the late 1950's, Dr. Breig was examining an encephalogram. He noticed that some clips that he had placed around the patient's cerebral blood vessels had moved between the pre- and post-operative pictures. He realized that this must be a representation of the movements of the brain, since blood vessels and neural tissues are anatomically connected [50].

Louis discovered in a cadaver study that the spinal canal undergoes considerable changes in length between the extremes of flexion and extension (slump test) [50]. The total change is of the order of 5-7 cm and is greater on the posterior than the anterior side. Similarly, with lateral flexion, the canal is lengthened on the convex side and shortened on the concave. He demonstrated the directions of neuro-meningeal movement in relation to the spinal canal mechanical interface.

\section{Treatment of the filum terminale}

Release of cord tethering, thereby allowing the spinal cord to resume normal tension, is the basis of the surgical treatment in patients with tethered spinal cord symptomatology. Recent studies have pointed out that if untethering is performed before there is irreversible neurological damage, improvement in motor and sensory function can be achieved.

The immediate improvement of symptoms in some patients with syringomyelia after sectioning of the filum terminale may be attributed to relief of traction and compression of the pericavitary medullary tissue, improving centrospinal ischemia and the tumour-like effect of the tractioned syringomyelia cavity [23]. Alf Breig, neurosurgeon, was the first to describe the mechanics in the spinal cord (neurodynamics): 'note that neural tissue moves in relation to dura and that dura moves in relation to canal. Tension changes are evident in the shape of the blood vessels [50].

Treatment of neurological aspects of the adolescent idiopathic scoliosis

The purpose of treating the brain in scoliosis is to balance the right and left hemispheres. Stress causes a imbalance, which may be the cause of the scoliosis. The osteopath uses an induction technique through the balance point fulcrum shift principle to balance the right and left hemispheres with each other. A compression of the third ventricle, balances the ergotrope with the trophotrope system and balances the hypothalamus. Corrections of the thoracic spine and the paravertebral chain affect the activity of the peripheral sympathetic nervous system. The hypothalamus also affects 
the functioning of the endocrine system. This has been shown to have an asymmetrical sensitivity to certain hormones such as leptin in AIS [40].

Special attention must be paid to the double-triad. The double-triad is formed by interaction between the eye, the balancing body and the high neck. We speak of a double-triad because all components are both motor and sensory. Dysfunctions at one or more parts of the triad disturb the regulation of the postural system with asymmetrical muscular regulation of the spine and a scoliosis as a possible result.

The control and treatment of the midlines should not be lacking in any approach to a scoliosis. Although there is not yet a clear one-way definition of the various midlines, the following structures should be examined and, when necessary, treated: the primary midline located at the level of the chorda dorsalis and where the axial skeleton develops: the dorsal midline: the central nervous system with its dural system and the ventral midline: a line that runs from the top of the skull over the ventral side of the body and ends at the height of the coccyx. All three midlines should be corrected as they may be the cause of a scoliosis and can best be treated in the order described above.

The treatment of the epiphysis is to normalize the high thoracic spinal cord segments in the case of a dysfunction, normalizing the ganglion cervical superior because of the sympathetic regulation of the epiphysis and normalizing the balance between right and left hemisphere. Remarkably, the epiphysis is the only unpaired nucleus in the brain and is located on the midline. Dysfunctions of the epiphysis directly affect scoliosis [40].

\section{Treatment of the circulatory aspects}

According to Mitchell, an important technique in the treatment of a scoliosis is the spinal fluid drive. Both temporals are fixed in external rotation and the occiput puts an emphasis on the extension phase in which CSF is "pushed" into the spinal channel. Studies of Grimes, among others, show that CSF circulation plays a role in the development of AIS [29].

Like the membrane system, the arterial system in the body is also like a "reciprocal tension" system. This means that the heart is in the centre of a web formed by the arteries. Disturbance of the tension of the arteries disrupts this reciprocal system and can cause a scoliosis. The osteopath corrects the reciprocal tension on the arterial system by a balance point fulcrum shift technique.
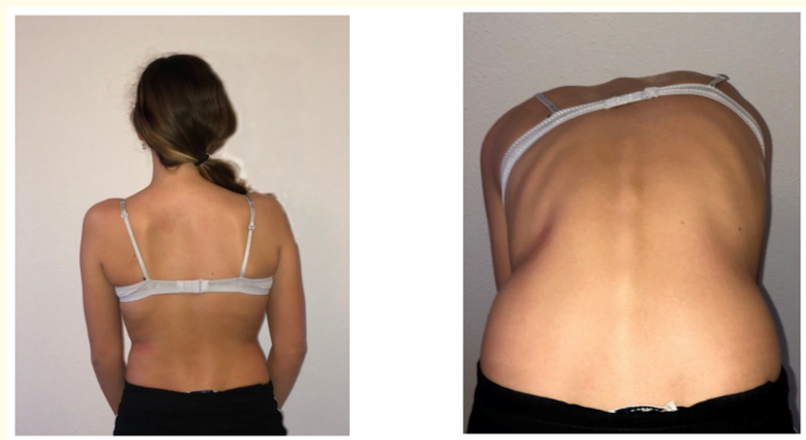

Figure 7: 13 year old girl with specific AIS. Right convexity with the apex around T5. Also notice the low amount of bodyfat and the absence of the normal Thoracic Kyphosis. (photo of Rene Zweedijk).

\section{Conclusion}

A. T. Still's aim was to improve the inherent self-healing mechanism of the body. W. G. Sutherland linked to this the importance of the cranium and its content. More recent insights define osteopathy by using the model of 5 . An osteopath should analyse the patient's health by using these 5 models. In the last decade, epigenetic aspects of many diseases have been described. Dysfunctions in one or more of the models might influence epigenetics transcription and can lead to disease. Chaos theory and complex systems theory make it clear that big changes in a chaordic organisation can take place very quickly, as clearly can be seen in the onset of AIS. The development of AIS is often very fast. Furthermore, one must realize that there are many possible causes. Scoliosis is always purely individual (every patient is different) and it is a dynamic process. There is an abundance of studies, which show that the aetiology of scoliosis, especially the aetiology of AIS, is complex and multi-factorial. The development of scoliosis is often sudden and "explosive". This supports the idea that a scoliosis must be seen as a reaction of a chaotic and complex adaptive system, epigenetic factors being triggered by several different events. The model of 5 supports the statement that the aetiology of scoliosis is multi-factorial. The osteopath should carefully study all of these aspects by keeping in mind 
the model of 5 and start the treatment as early as possible before the scoliosis becomes structural.

\section{Bibliography}

1. Mordecai SC and HV Dabke. "Efficacy of exercise therapy for the treatment of adolescent idiopathic scoliosis: a review of the literature". European Spine Journal 21.3 (2012): 382-389.

2. Menger RP and AH Sin. "Adolescent and Idiopathic Scoliosis, in StatPearls". Treasure Island (FL) (2019).

3. Shakil H., et al. "Scoliosis: review of types of curves, etiological theories and conservative treatment". Journal of Back and Musculoskeletal Rehabilitation 27.2 (2014): 111-115.

4. Chila A. "Foundations of Osteopathic Medicine" (2012).

5. Ward RC and RJ Hruby. "Foundations for osteopathic medicine". Philadelphia, Pa.: Lippincott Williams and Wilkins (2003).

6. Magoun HI. F. Sutherland Cranial Teaching, and A. Osteopathic Cranial, Osteopathy in the cranial field. [Fort Worth, Tex.]: Sutherland Cranial Teaching Foundation (1997).

7. Barral JP and P Mercier. "Visceral manipulation". Seattle: Eastland Press (2005).

8. Sarnadskiy VN. "Classification of postural disorders and spinal deformities in the three dimensions according to computer optical topography". Studies in Health Technology and Informatics 176 (2012): 159-163.

9. Anderson SM. "Spinal curves and scoliosis". Radiologic Technology 79.1 (2007): 44-65.

10. Wang J., et al. "Measurement of scoliosis Cobb angle by end vertebra tilt angle method". Journal of Orthopaedic Surgery and Research 13.1 (2018): 223.

11. Hruby RJ., et al. "The five osteopathic models: rationale, application, integration: from an evidence-based to a person-centered osteopathy". (2017).

12. Sergueef N., et al. "Palpatory diagnosis of plagiocephaly". Complementary Therapies in Clinical Practice 12.2 (2006): 101-110.

13. Lessard S., et al. "Exploring the impact of osteopathic treatment on cranial asymmetries associated with nonsynostotic plagiocephaly in infants". Complementary Therapies in Clinical Practice 17.4 (2011): 193-198.

14. Nakashima A., et al. "The relationship between lateral displacement of the mandible and scoliosis". Journal of Oral and Maxillofacial Surgery 21.1 (2017): 59-63.

15. Kim TH., et al. "The relation between idiopathic scoliosis and the frontal and lateral facial form". Korean Journal of Orthodontics 44.4 (2014): 254-62.

16. Roth M. "Idiopathic scoliosis caused by a short spinal cord". Acta Radiologica (Stockh) 7.3 (1968): 257-271.

17. Porter RW. "Can a short spinal cord produce scoliosis?". European Spine Journal 10.1 (2001): 2-9.

18. Guo X., et al. "Relative anterior spinal overgrowth in adolescent idiopathic scoliosis. Results of disproportionate endochondralmembranous bone growth". The Journal of Bone and Joint Surgery British 85.7 (2003): 1026-1031.

19. Chu WC., et al. "Relative shortening and functional tethering of spinal cord in adolescent idiopathic scoliosis?: study with multiplanar reformat magnetic resonance imaging and somatosensory evoked potential". Spine (Phila Pa 1976) 31.1 (2006): E19-25.

20. Hefti F. "Pathogenesis and biomechanics of adolescent idiopathic scoliosis (AIS)". Journal of Children's Orthopaedics 7.1 (2013): 17-24.

21. De Vloo P., et al. "The Filum Terminale: A Cadaver Study of Anatomy, Histology, and Elastic Properties". World Neurosurgery 90 (2016): 565-573 e1.

22. Royo-Salvador MB., et al. "Results of the section of the filum terminale in 20 patients with syringomyelia, scoliosis and Chiari malformation". Acta Neurochira (Wien), 147.5 (2005): 515-523.

23. Royo-Salvador MB., et al. "The Filum disease and the NeuroCranio-vertebral syndrome: definition, clinical picture and imaging features". BMC Neurology 20.1 (2020): 175.

24. Faloon M., et al. "Incidence of Neuraxial Abnormalities Is Approximately 8\% Among Patients With Adolescent Idiopathic Scoliosis: A Meta-analysis". Clinical Orthopaedics and Related Research 476.7 (2018): 1506-1513. 
25. Craig AD. "Forebrain emotional asymmetry: a neuroanatomical basis?" Trends in Cognitive Sciences 9.12 (2005): 566-571.

26. Burwell RG., et al. "Adolescent idiopathic scoliosis (AIS), environment, exposome and epigenetics: a molecular perspective of postnatal normal spinal growth and the etiopathogenesis of AIS with consideration of a network approach and possible implications for medical therapy". Scoliosis 6.1 (2011): 26.

27. Burwell RG., et al. "Pathogenesis of adolescent idiopathic scoliosis in girls - a double neuro-osseous theory involving disharmony between two nervous systems, somatic and autonomic expressed in the spine and trunk: possible dependency on sympathetic nervous system and hormones with implications for medical therapy". Scoliosis 4 (2009): 24.

28. Morningstar MW., et al. "Chiropractic Treatments for Idiopathic Scoliosis: A Narrative Review Based on SOSORT Outcome Criteria". Journal of Chiropractic Medicine 16.1 (2017): 64-71.

29. Grimes DT., et al. "Zebrafish models of idiopathic scoliosis link cerebrospinal fluid flow defects to spine curvature". Science 352.6291 (2016): 1341-1344.

30. Talic G., et al. "The Effect of Adolescent Idiopathic Scoliosis on the Occurrence of Varicose Veins on Lower Extremities". Medical Archives 71.2 (2017): 107-109.

31. Grant CA., et al. "A comparison of vertebral venous networks in adolescent idiopathic scoliosis patients and healthy controls". Surgical and Radiologic Anatomy 39.3 (2017): 281-291.

32. Ratcliffe JF. "An evaluation of the intra-osseous arterial anastomoses in the human vertebral body at different ages. A microarteriographic study". Journal of Anatomy 134 (1982): 373382.

33. Jiang, H., et al. "The position of the aorta changes with altered body position in single right thoracic adolescent idiopathic scoliosis: a magnetic resonance imaging study". Spine (Phila Pa 1976) 37.17 (2012): E1054-1061.

34. Kaila R., et al. "Scoliosis development in identical twins after intercostal thoracotomy for pulmonary artery sling correction". Annals of the Royal College of Surgeons of England 88.6 (2006): W1-3.
35. Sevastik J., et al. "A new concept for the etiopathogenesis of the thoracospinal deformity of idiopathic scoliosis: summary of an electronic focus group debate of the IBSE". European Spine Journal 12.4 (2003): 440-450.

36. Levillain A., et al. "Short-term foetal immobility temporally and progressively affects chick spinal curvature and anatomy and rib development". European Cell Material 37 (2019): 23-41.

37. Zaydman AM., et al. "A New Look at Etiological Factors of Idiopathic Scoliosis: Neural Crest Cells". International Journal of Medical Sciences 15.5 (2018): 436-446.

38. Machida, M., et al. "Melatonin. A possible role in pathogenesis of adolescent idiopathic scoliosis". Spine (Phila Pa 1976) 21.10 (1996): 1147-1152.

39. Ahuja K., et al. "A Comparative Analysis of the Metabolic and Coagulative Profiles in Patients with Idiopathic Scoliosis, Congenital Scoliosis and Healthy Controls: A Case-Control Study". Asian Spine Journal 12.6 (2018): 1028-1036.

40. Fadzan M and J Bettany-Saltikov. "Etiological Theories of Adolescent Idiopathic Scoliosis: Past and Present". Open Orthopadic Journal 11 (2017): 1466-1489.

41. Shen N., et al. "Alterations of the gut microbiome and plasma proteome in Chinese patients with adolescent idiopathic scoliosis". Bone 120 (2019): 364-370.

42. Zaina F., et al. "Prevalence and predictors of adolescent idiopathic scoliosis in adolescent ballet dancers". Archives of Physical Medicine and Rehabilitation 96.6 (2015): 1181.

43. Ogura Y., et al. "An international meta-analysis confirms the association of BNC2 with adolescent idiopathic scoliosis". Science Report 8.1 (2018): 4730.

44. Kikanloo SR., et al. "Etiology of Adolescent Idiopathic Scoliosis: A Literature Review". Asian Spine Journal 13.3 (2019): 519-526.

45. Meng Y., et al. "Value of DNA methylation in predicting curve progression in patients with adolescent idiopathic scoliosis". EBioMedicine 36 (2018): 489-496.

46. Ogura Y., et al. "Epigenetics for curve progression of adolescent idiopathic scoliosis". EBioMedicine 37 (2013): 36-37. 
47. Taylor-Swanson L., et al. "Complex Adaptive Systems Theory and Inter-Rater Reliability: Proposed Answers to Challenging Questions". Journal of Alternative and Complementary Medicine 25.11 (2019): 1074-1076.

48. Lotan S and L Kalichman. "Manual therapy treatment for adolescent idiopathic scoliosis". Journal of Bodywork and Movement Therapies 23.1 (2019): 189-193.

49. Schwind P., et al. Praxishandbuch Faszienbehandlung: Muskelfaszien, Membranen, Organhüllen (2018).

50. Breig A. "Adverse mechanical tension in the central nervous system: an analysis of cause and effect: relief by functional neurosurgery". Stockholm: Almqvist and Wiksell (1978).

\section{Assets from publication with us}

- Prompt Acknowledgement after receiving the article

- Thorough Double blinded peer review

- Rapid Publication

- Issue of Publication Certificate

- High visibility of your Published work

Website: $\underline{w w w}$.actascientific.com/

Submit Article: www.actascientific.com/submission.php

Email us: editor@actascientific.com

Contact us: +919182824667 